\title{
DEVELOPING AFFORDABLE BATHYMETRIC ANALYSIS TECHNIQUES USING NON-CONVENTIONAL PAYLOAD FOR CULTURAL HERITAGE INSPECTIONS
}

\author{
F. Mugnai ${ }^{1,2, *}$ A. Ridolfi ${ }^{3}$, M. Bianchi ${ }^{3}$, M. Franchi $^{3}$, G. Tucci $^{1}$ \\ ${ }^{1}$ Department of Civil and Environmental Engineering, University of Florence, Florence, Italy - francesco.mugnai@ec.europa.eu \\ ${ }^{2}$ European Commission, Joint Research Centre (JRC), Directorate A - Scientific Development Unit \\ ${ }^{3}$ Department of Industrial Engineering, University of Florence, Florence, Italy
}

Commission II, WG II/8

KEY WORDS: Autonomous Underwater Vehicles, Bathymetric Surveys, Acoustic Devices, Cultural Heritage inspections

\begin{abstract}
:
This paper focuses on the implementation of new techniques for bathymetric inspections. The scope is the exploitation of sensors, usually and commonly used for navigation, namely the altimeter and the Forward Looking Sonar (FLS), for identifying objects which are laying on the sea floor. In this particular framework, the low spatial resolution and coverage of these sensors have been enhanced through the application of classical computational geometry. The altimeter and the FLS are part of the most common underwater navigation systems, and they are vastly mounted on Autonomous Underwater Vehicles. Although they are not designed for this kind of accurate measurements and for 3D spatial reconstruction, they are quite cost-effective if compared with standard multibeam acoustic systems. Developing a technique for exploiting such an affordable low cost and widely used sensor will empower the Cultural Heritage community of users, giving a feasible opportunity to perform effective archaeological campaigns also within small funded projects.
\end{abstract}

\section{INTRODUCTION}

Within a scenario in which archaeologists and managers of underwater and coastal cultural heritage perform complex and totally different tasks one to each other, e.g., investigations, monitoring, and safeguarding as well as risk management, site documentation, and site management (to name a few), these figures dealing with underwater and coastal cultural heritage are always open to apply new innovating technologies. In particular, taking into account what stated in the Manual for Activities directed at Underwater Cultural Heritage (Maarleveld et al., 2013), "the underwater archaeological survey (pre-disturbance survey and site monitoring survey) comprises the process of locating, exploring and recording a site. Its aims and objectives are determined in the project design, thus the survey is an end point in itself", the utility of innovative survey techniques holds surely a position of chief-point.

Even though the practical field work carried out by underwater archaeologists cannot be totally replaced, advances in marine and terrestrial scanning survey techniques, together with increasingly more precise inertial navigation sensors, multisensor acquisition system and cost-effective Unmanned Aerial Systems (UAS) photogrammetry allow hydrographic surveyors to undertake high-precision marine asset integrity mapping (Ramsay et al., 2016). Current approaches adopted by underwater archaeologists mainly consist of offset measurements, tape measure trilateration and simple photography (Van Damme, 2015). In addition to the intrinsic safety matters related to the direct involvement of human operators in the data capture process in a potentially dangerous environment, some of the tasks performed by archaeologists typically suffer from time consuming issues and relevant risk of human errors within the possible hostile working scenario. In the past and also currently, many archaeological campaigns make use of divers

\footnotetext{
${ }^{*}$ Corresponding author
}

and Remotely Operated Vehicles (ROVs). ROVs and different tools have been developed to help archaeologists work (Sorbi et al., 2008). Autonomous Underwater Vehicles (AUVs) can be considered as a natural evolution of the ROVs. Using reliable AUVs can strongly help in reducing the costs of the logistics (e.g., dedicated personnel piloting the vehicle is not needed, differently with respect to ROVs, and the support vessel either). Detailed knowledge of underwater topography, is crucial for many research fields. In archaeology, it is the basis for understanding of organization and distribution of archaeological sites along and within water bodies (Doneus et al., 2013).

During the last decades, several methods to perform seabed topography have been proposed, such as airborne/surface-based solutions, and multibeam-based ones; all of them are known to provide valuable large scale terrain maps of the sea floor (Bingham et al., 2010). On the other hand, high costs can lead to limited inspections and surveys, thus posing a constraint to the seabed coverage. Small projects, archeological communities of users and other research activities are above all penalized. Broadly speaking, AUVs are becoming effective tools for locating, identifying, and surveying archaeological sites (Roman, Mather, 2010).

\section{MAIN CONTRIBUTION}

The logistics of an underwater archaeological research often appears to be quite complicated in comparison to the terrestrial field research. The application of all the methods able to reduce the time spent underwater, the amount of fieldwork required during an underwater survey, and also the cost of the overall missions could thus lead to significant efficiency improvements and cost savings.

The research work carried out and presented in this paper aims to tackle the issues related to the bathymetric surveys for cultural heritage inspections. In fact, the high quality and resol- 
ution of the bathymetric maps play a key role both in archaeological discoveries of new sites and in monitoring the ones already found. Unfortunately, on the other hand, bathymetric sensors (especially when mounted on AUVs) involve high costs and their exploitation should be limited to those cases of actual necessity or restricted to specific areas. In this framework, the investigation of an innovative and cost effective approach that allows to quickly detect those areas (by the identifications of possible objects of interest lying on the sea-bottom) where a further investigation, i.e., a bathymetric survey, is needed could lead to significantly reduce the cost related to it. In fact, the possibility of performing a preliminary affordable bathymetric analysis exploiting common navigation sensors mounted on AUVs yields to restrict the areas to be inspected by the use of bathymetric sensors and therefore limits its exploitation (and cost) only in a defined space.

With respect to the current state-of-the-art methods, the one here proposed does not present, as reported in Sec. 5, an alternative to the already available solutions but it strives to become a good practice to be used before performing a bathymetry to reduce the cost of such a survey.

\section{METHODOLOGY}

To investigate potential and effectiveness of the proposed technique, tests within a controlled environment have been carried out. In particular, two different test campaigns have been executed, each one assessing the exploitation of different sensors that can be considered "non-conventional" for bathymetric purposes, but that are common payload to navigate.

\subsection{Bathymetry with punctual altimeter measurements}

During the first tests, a basin in the NATO Centre for Maritime Research and Experimentation (CMRE), La Spezia (Italy), has been used as test site (basin of the ERL/SAUC-e 2018 European Competition $^{1}$ ). The scope was to acquire a series of altitude measurements of the basin floor through an altimeter integrated within a Doppler Velocity Log (DVL) sensor. Afterwards, all the above mentioned measurements have been interpolated and smoothed. A target, namely some empty plastic cylinders (Fig. 2), has been positioned on the basin floor and represented a submerged site to be detected. It is useful underlying that the DVL sensor, that has been installed on FeelHippo AUV ${ }^{2}$ (Fig. 1) (Allotta et al., 2017), uses a long pulse along three acoustic beams, each pointing in a different direction, to measure multiple distances and to obtained the vehicle altitude (namely its vertical distance from the sea bottom). Interpolation and smoothing have been performed through computing Delaunay Triangulation (Delaunay, 1934): for a given set $\mathrm{P}$ of discrete points in a plane is a triangulation $\mathrm{DT}(\mathrm{P})$ such that no point in $\mathrm{P}$ is inside the circumcircle of any triangle in DT(P). In particular, FeelHippo AUV performed several missions with designed lawnmower paths and both altitude values (provided by the altimeter integrated in the DVL) and vehicle (absolute) position have been acquired. Afterwards, offline 3D meshes have been separately elaborated from each mission (Fig. 3). Lastly, by applying Bowyer Watson algorithm (Rebay, 1993) an augmented dataset constituted by the data gathered during the different

\footnotetext{
${ }^{1}$ https://eu-robotics.net/robotics_league/ erl-emergency/about/index.html, visited June 2019

${ }^{2}$ Autonomous underwater vehicle that has been developed by the University of Florence (Department of Industrial Engineering).
}

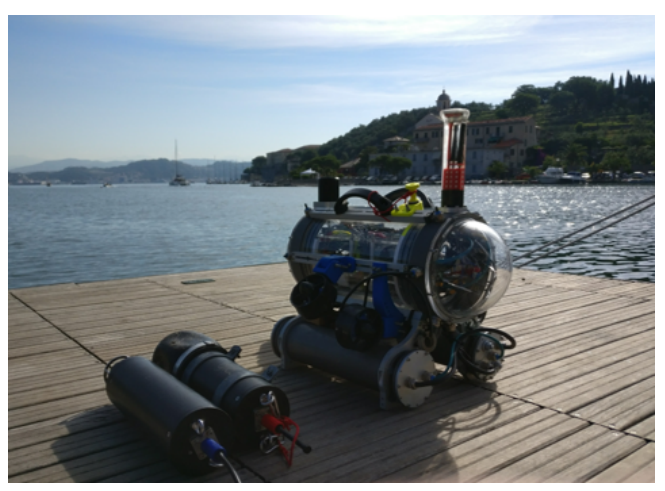

Figure 1. FeelHippo AUV designed and built by the Department of Industrial Engineering of Florence

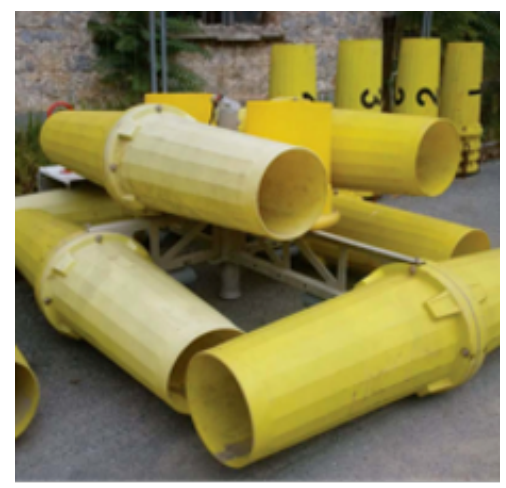

Figure 2. Empty plastic cylinders representing a submerged structure

underwater missions, has been tested (Fig. 3). The final result, which can draw to an increased number of altitude points, obviously presents a more precise $3 \mathrm{D}$ mesh.

\subsection{Bathymetry with Forward Looking Sonar}

As reported at the beginning of Sec. 3, a second test campaign has been carried out to evaluate the possibility of using another sensor not usually used for bathymetry reasons. In particular, with the aim of investigating the use of a Forward Looking Sonar (FLS) for bathymetry purposes, an additional test campaign was performed at Marina di Pisa, Pisa (Italy) in July 2018. The goal was to obtain a series of altitude measurements in the coastal area close to the Arno River mouth exploiting data gathered from a BlueView M900 FLS by Teledyne. It is worth pointing out that, in this occasion, the FLS was mounted approximately perpendicular with respect to the sea-bottom (in other words with a tilt angle close to $90^{\circ}$ ). In this particular configuration, the FLS is able to deal with a wider area that spans along its azimuth angle (in our case $130^{\circ}$ ). The main advantage is the possibility to cope with a greater number of sampled points for each acquisition, leading to a more dense point cloud but, at the same time, leaving unaltered interpolation and smoothing process. Therefore, for the sake of brevity, the above mentioned Delaunay Triangulation technique as well the Bowyer Watson algorithm are not discussed again.

On the other hand, although the covered area results largely increased without any addition in the performed underwater mission, the altitude accuracy gets slightly poorer with respect to the above mentioned altimeter-based method (from few centimeters to ten-twenty centimeters). Obviously, the overall ac- 

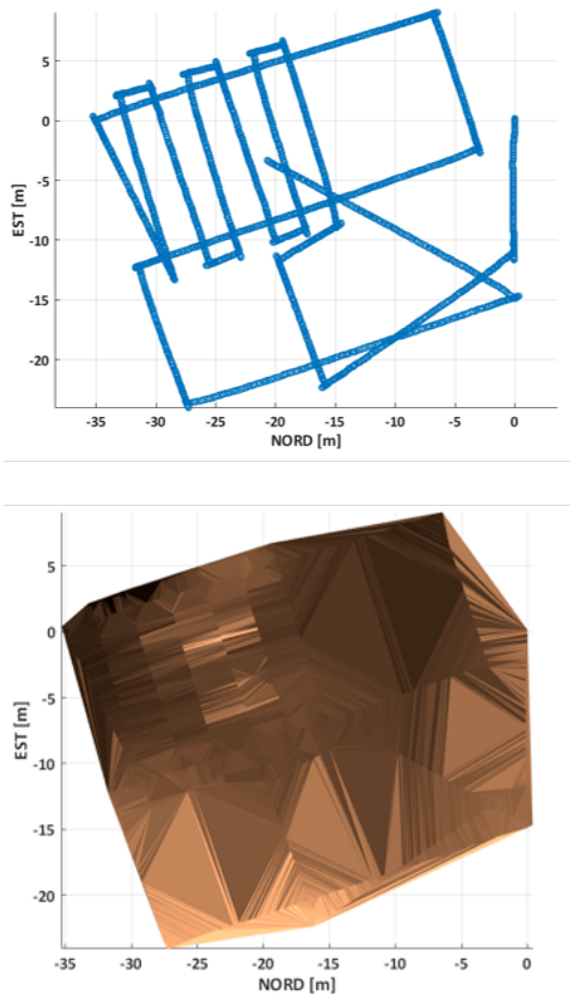

Figure 3. First recorded path (top) and original 3D mesh (bottom)

curacy of the bathymetric map is affected by the navigation and localization performance of the underwater vehicle.

\section{RESULTS}

Because of the lower density of the point cloud (reproducing the sea-bottom bathymetry) generated by the use of the altimeter with respect to the exploitation of the FLS, in this section, the first method will be assessed in terms of possibility of detecting submerged object of interests, while the FLS-based strategy is reported only as an additional (more accurate) solution that can be adopted.

3D models of sea bed are produced. The 3D mesh, made through measurements performed by the altimeter integrated within the DVL sensor during a single AUV mission has still a low spatial resolution, if compared with point clouds from multibeam or laser scanning tools. However, by applying Bowyer Watson algorithm, the accuracy of 3D mesh is remarkably improved by adding new points to the original 3D mesh (Fig. 4) and the localization of specific targets results feasible (Fig. 5).

In addition to this, a FLS-based bathymetry method is described and its pros and cons, with respect to an altimeter-based one, are discussed and the final results are presented in Fig. 6. It is worth stressing that the above mentioned methods present the same post-processing operations (namely Delaunay technique and the Bowyer Watson method for triangulation and smoothing), differing only from the acquisition device.

\section{CONCLUSIONS}

The proposed and described research activity aimed to define a low-cost methodology capable of, by the use of sensors com- https://www.overleaf.com/project/5d124c873b99c86f0b457f78
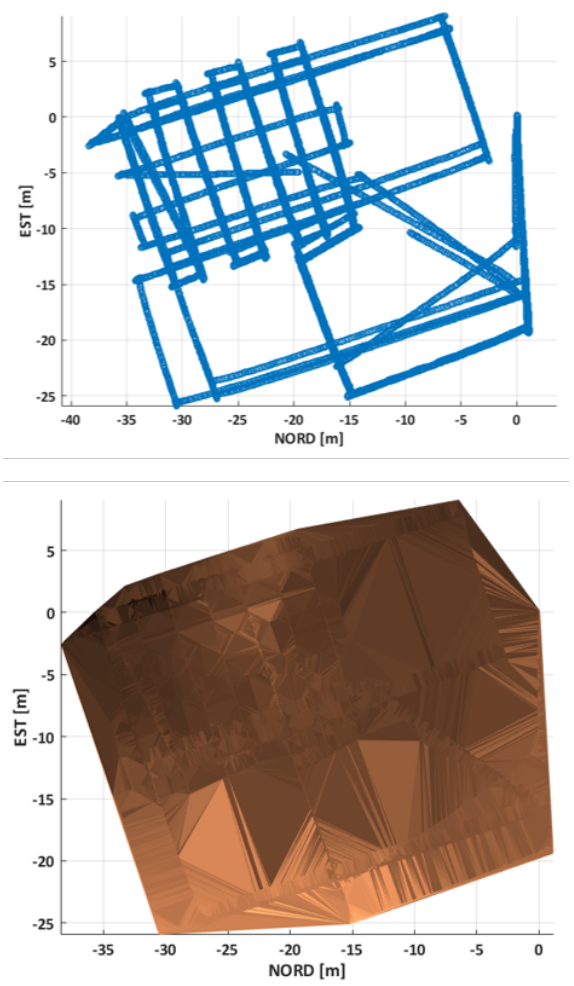

Figure 4. Multiple recorded paths (top) and 3D mesh from BW algorithm application (bottom)

monly mounted on AUVs for navigation, detecting objects of interest and consequently defying specific areas where planning a further inspection using more accurate sensors, i.e., bathymetric payload.

Although the resolution of the 3D mesh created from multiple paths dataset is still too low to perform reliable archaeological inspections, exploiting a side-capability of a navigation sensor (namely the altimeter included in a DVL already added to the vehicle to allow for an accurate navigation and a FLS usually adopted during underwater missions) has given appreciable results that foster further investigations. As a matter of facts, although an accurate reconstruction of targets shape is not feasible by analyzing the reconstructed 3D meshes, localizing targets is definitely possible with the use of the information gathered by the use of the altimeter (Fig. 5).

Moreover, with the aim of enhancing the generated points cloud, a FLS-based bathymetry method is proposed. At the expanse of a slightly worse altitude accuracy, the described solution is able to limit the time necessary to obtain a full coverage of the inspection site, avoiding time-consuming operations.

As conclusion, the proposed methods show a complementary behaviour in terms of accuracy and coverage, shedding light on a non-conventional and low-cost solution for cultural heritage inspections.

Future developments will involve the testing of the two strategies together, proposing a complete framework able to cover areas of the order of hundreds of square meters with an acceptable accuracy and without performing long timeconsuming underwater missions. 


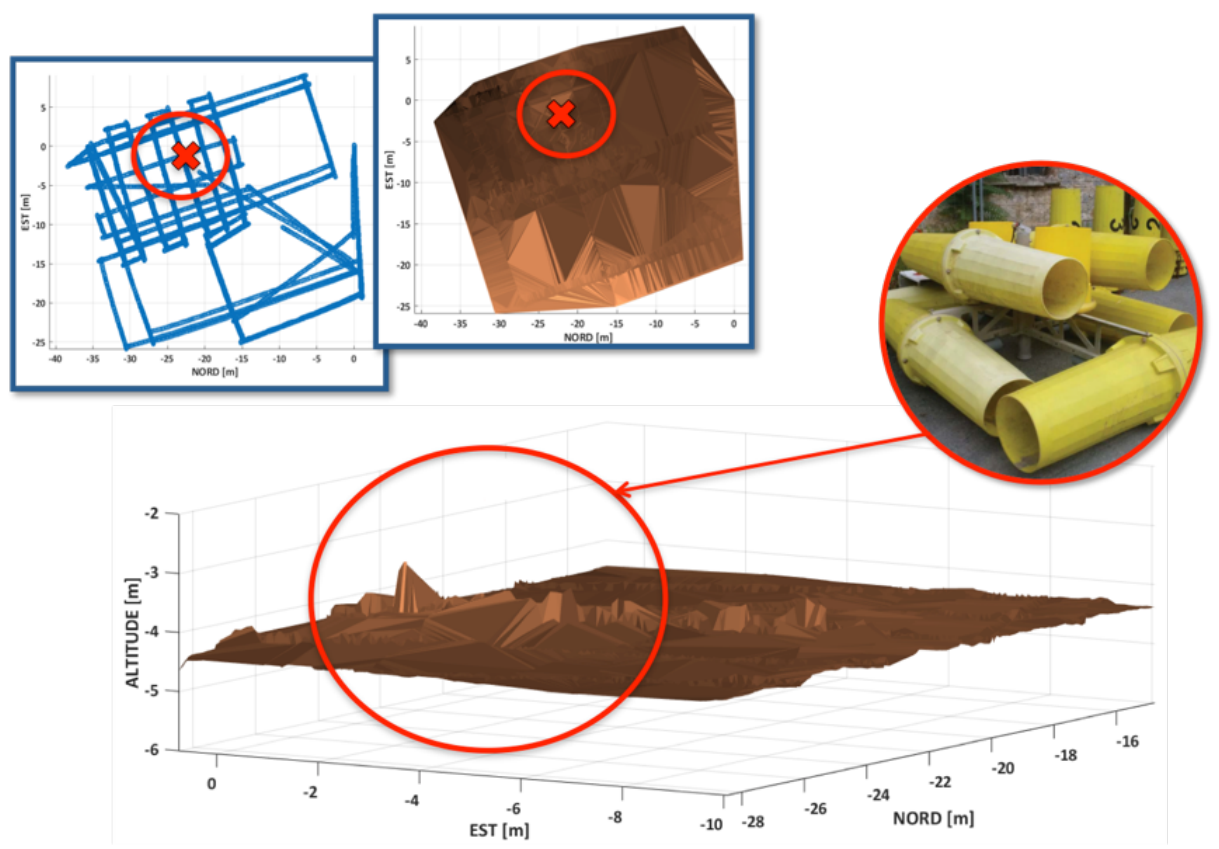

Figure 5. Localization of targets on the final 3D mesh

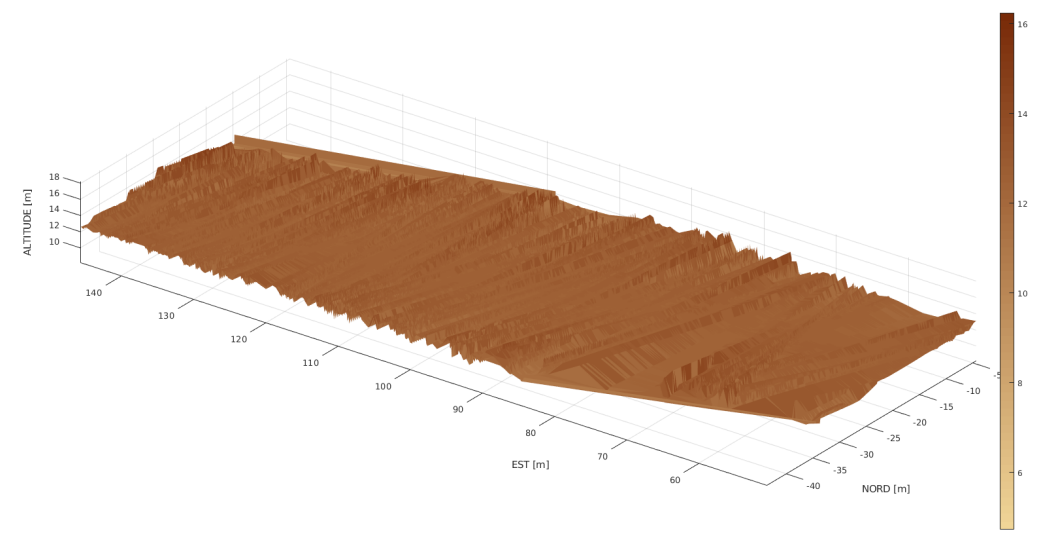

Figure 6. The underwater bathymetry map of the coastal area close to the Arno River mouth.

\section{ACKNOWLEDGEMENTS}

A special thank goes to Rosalinda Verzino and Alessandra Capurro who collaborated to this work during their Bachelors theses and to the UNIFI Robotics Team who attended the ERL/SAUCe 2018 European Competition with FeelHippo AUV, reaching the first prize.

\section{REFERENCES}

Allotta, B., Conti, R., Costanzi, R., Fanelli, F., Gelli, J., Meli, E., Monni, N., Ridolfi, A., Rindi, A., 2017. A low cost autonomous underwater vehicle for patrolling and monitoring. Proceedings of the Institution of Mechanical Engineers, Part M: Journal of Engineering for the Maritime Environment, 231(3), 740-749.

Bingham, B., Foley, B., Singh, H., Camilli, R., Delaporta, K., Eustice, R., Mallios, A., Mindell, D., Roman, C., Sakellariou,
D., 2010. Robotic tools for deep water archaeology: Surveying an ancient shipwreck with an autonomous underwater vehicle. Journal of Field Robotics, 27(6), 702-717. https://onlinelibrary.wiley.com/doi/abs/10.1002/rob.20350.

Delaunay, B., 1934. Sur la sphre vide. Bulletin de lÁcadmie des Sciences de lÚRSS, Classe des sciences mathmatiques et naturelles, 6, 793-800.

Doneus, M., Doneus, N., Briese, C., Pregesbauer, M., Mandlburger, G., Verhoeven, G., 2013. Airborne laser bathymetry detecting and recording submerged archaeological sites from the air. Journal of Archaeological Science, 40(4), 2136 2151.

Maarleveld, T. J., Guérin, U., Egger, B., 2013. Manual for Activities directed at Underwater Cultural Heritage: guidelines to the Annex of the UNESCO 2001 Convention. Unesco.

Ramsay, P., Murrell, D., Wilkinson, D., 2016. Using multibeam bathymetry, topographic laser scanning \& UAS photo- 
grammetry: Marine asset integrity mapping. Hydro International, 20, 24-26.

Rebay, S., 1993. Efficient Unstructured Mesh Generation by Means of Delaunay Triangulation and Bowyer-Watson Algorithms. Journal of Computational Physics, 106(1).

Roman, C., Mather, R., 2010. Autonomous Underwater Vehicles as Tools for Deep-Submergence Archaeology. Proceedings of the Institution of Mechanical Engineers, Part M: Journal of Engineering for the Maritime Environment, 224(4), 327-340.

Sorbi, L. et al., 2008. Robotic tools and techniques for improving research in an underwater delicate environment. Marine Technology Society Journal, 49(5), 6-17.

Van Damme, T., 2015. Computer Vision Photogrammetry for Underwater Archaeological Site Recording in a Low-Visibility Environment. ISPRS - International Archives of the Photogrammetry, Remote Sensing and Spatial Information Sciences, 231238. 